\title{
Repair of Olecranon Fracture in a Dog with Pinning and Tension Band Wiring Techniques First Case Report in Bangladesh
}

\section{Thomby Paul, Sreekanta Biswas, Sabiha Zarin Tasnim Bristi, Debashish Sarker, Saroj Kumar Yadav and Bhajan Chandra Das*}

Department of Medicine and Surgery, Faculty of Veterinary Medicine, Chattogram Veterinary and Animal Sciences University, Bangladesh.

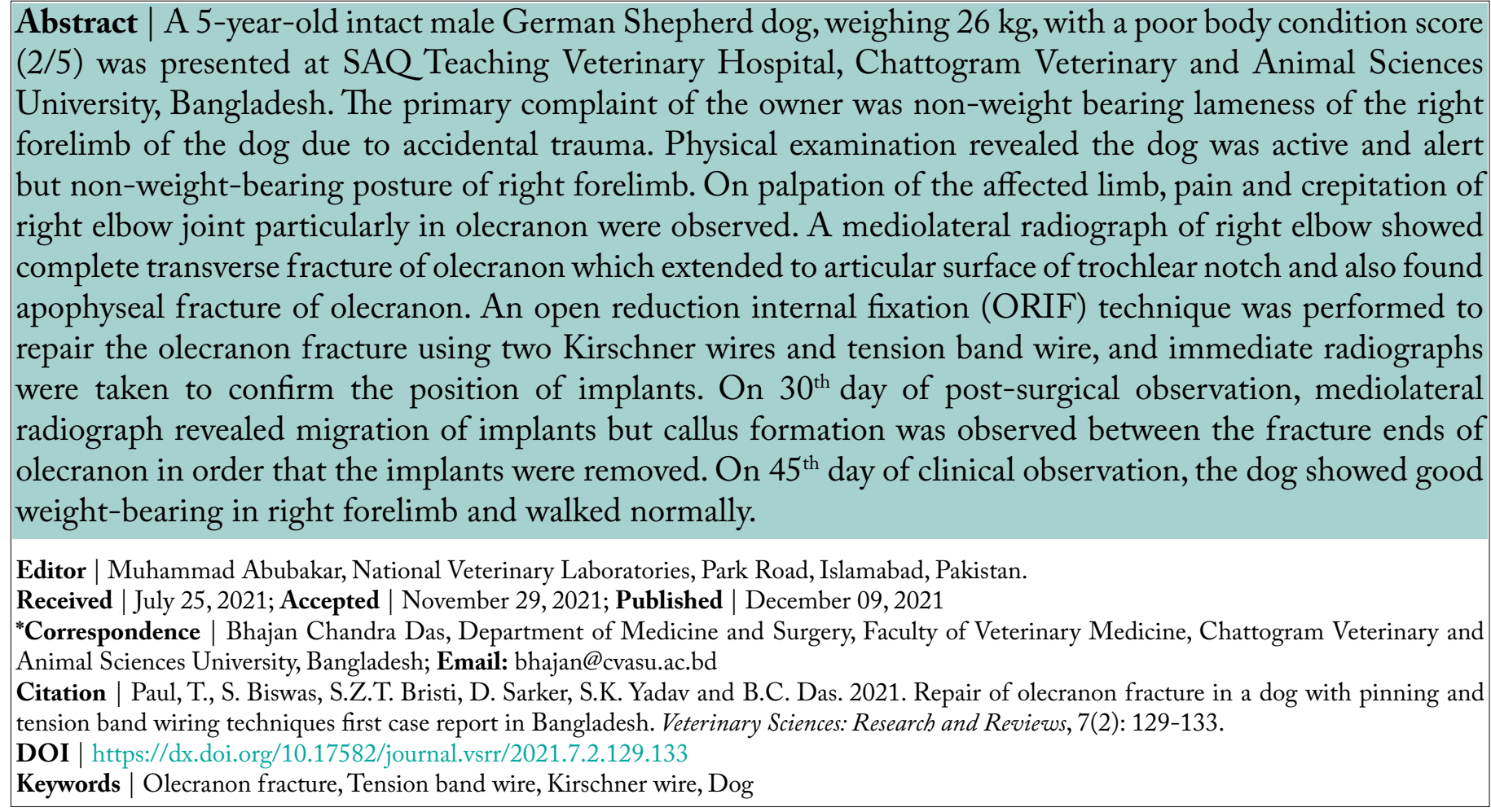

\section{Introduction}

$\mathrm{T}$ he Olecranon process is the most proximal part of ulnar bone which is comprised of olecranon tuber, anconeal process and proximal part of trochlear notch (Evans and de Lahunta, 2012). Triceps brachii muscle inserts on the olecranon process of ulnar bone which assists to extend the elbow joint and enable to bear weight of limb (Evans and de Lahunta, 2012). Olecranon fractures are uncommon in dogs but occasionally occur due to human trauma (Grafinger et al., 2007). The fractures of olecranon may be either extra-articular (proximal to the trochlear notch) or intra-articular surface through the trochlear notch (Nayak et al., 2012). These fractures are usually seen in young dogs and represent apophyseal separation of the olecranon (Newton and Nunamaker, 1985). But in older dogs, the fractures may be entered into elbow joint or may be comminuted, and the fracture segments of olecranon maybe displaced proximally due to the pull of triceps brachii muscle (Newton and Nunamaker, 1985). The fractures can be repaired 
through applying different techniques like as pinning and external fixation or tension-band wiring or combination of tension-band wiring and lag screw or combination of Kirschner wire (K-wire) and tensionband wiring (Mullett et al., 2000; Tyagi et al., 2002; Grafinger et al., 2007). Among them, tension-band wiring technique is commonly used in repair of olecranon fractures, and provide adequate stability as well as immediate weight-bearing (Nayak et al., 2012). The objective of the study was management of olecranon fracture by tension band wiring and pinning techniques.

\section{Material and Methods}

An intact male German Shepherd dog aged 5 years at $26 \mathrm{~kg}$ body weight with poor body condition score $(2 / 5)$ was brought to SAQTeaching Veterinary Hospital, Chattogram Veterinary and Animal Sciences University, Bangladesh. The dog had a history of nonweight bearing lameness of right forelimb since 3 days due to accidental trauma. Physical examination revealed the dog was active and alert but non weight bearing lameness of right forelimb (Figure 1). Rectal body temperature $\left(101^{\circ} \mathrm{F}\right)$, heart rate $(90 / \mathrm{min}$.) and respiration rate $(28 / \mathrm{min}$.) were normal; pink and moist mucous membranes with capillary refill time (CRT) was <2 second. On palpation of the affected limb, pain and crepitation of right elbow joint particularly in olecranon of ulnar bone were observed. A mediolateral radiograph of right elbow revealed transverse fracture of right olecranon which extended to articular surface of trochlear notch (Figure 2). Apophyseal fracture of olecranon was also found. Based on the clinical and radiographic findings, it was decided to perform tension band wiring and pinning for repairing of the olecranon fracture.

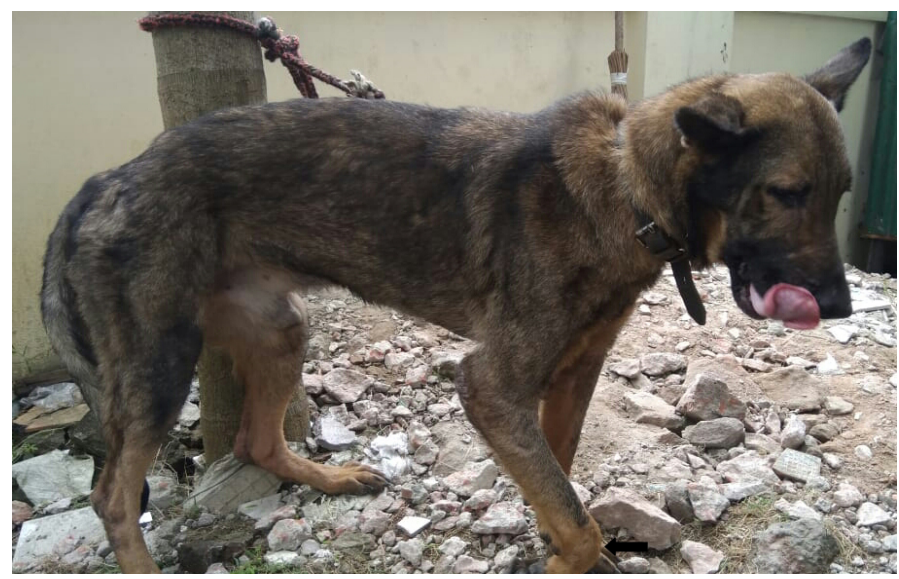

Figure 1: The dog was showing non-weight bearing posture of right forelimb (arrow).

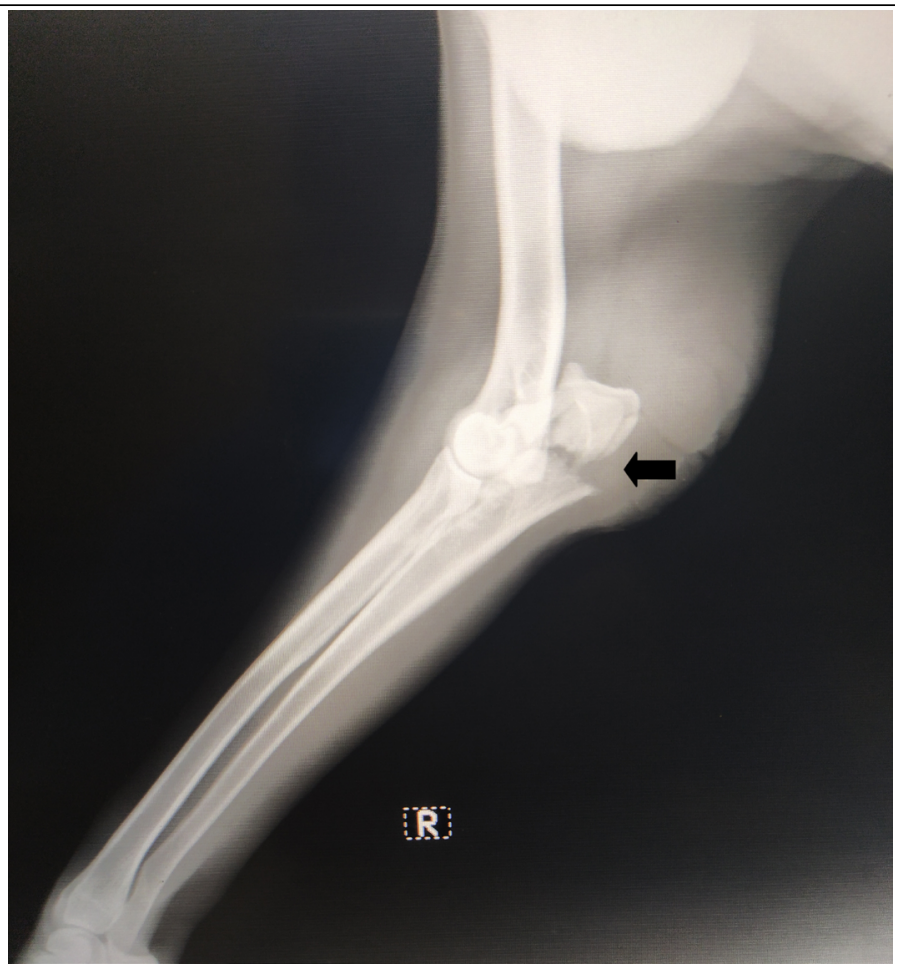

Figure 2: Preoperative mediolateral radiograph of right elbow revealed transverse fracture of olecranon (arrow).

The patient was fasted about 6 hours prior to surgery. Shaving was performed on surgical area and prepared the patient aseptically by using $7.5 \%$ providone iodine and $70 \%$ isopropyl alcohol. Xylazine hydrochloride was administered intramuscularly at the dose rate $1.0 \mathrm{mg} / \mathrm{kg}$ body weight as a pre-anesthetic agent. The patient was kept in left lateral recumbancy on operative table and normal saline $(0.9 \% \mathrm{NaCl})$ was infused intravenously at the rate of $10 \mathrm{ml} / \mathrm{kg} / \mathrm{hour}$. Ketamine hydrochloride was considered as general anaesthetic injecting intravenously at the dose rate $8.0 \mathrm{mg} / \mathrm{kg}$ body weight.

A linear incision was made on skin at caudal aspect of the olecranon after approaching the fractured site externally. Blunt incisions were made on subcutaneous tissue and fascia to expose the olecranon. The space between the fracture segments were reduced and brought to the apposition. Unfortunately, a small bony part from the proximal fracture end of the olecranon was separated. A 20 guage stainless wire was passed through middle of the separated part after drilling and was fixed with proximal fracture end of the olecranon at caudal aspect. Two kirschner wires (2 $\mathrm{mm}$ ) were inserted normograde from the olecranon tuber into the proximal diaphyseal medullary canal of the ulna. The protruded ends of the K-wires were bent caudally close to the olecranon tuber. The spare ends of $\mathrm{K}$-wires were cut leaving a short bent which 
formed a hook and rotated cranially. The same size of wire was used anchoring around the proximal hooks of the $\mathrm{K}$ - wires. In transverse direction, a $2.0 \mathrm{~mm}$ hole was drilled distal to the fractured end using drill bit and a free end of wire was passed through the holes. An eight loop figure was created by the wire over the tension surface. The both ends of the wire were twisted together and tightened using wire twister. The twisted wire was cut leaving the four twists. The muscle layers were apposed and sutured using polyglactin $1-0$ in a simple continuous pattern. The fascia and subcutaneous tissues were sutured using same suture materials and same pattern. Finally, the skin edges were sutured with cross mattress pattern by using non-absorbable suture materials. The surgical wound was covered by a sterile pad after application of topical ointment of povidone iodine. Modified Robert Jones bandage was applied in the affected limb after operation for immobilization of the joint up to 4 weeks. Mediolateral and craniocaudal radiographs of right elbow were taken just after fixation of fractured segments of the olecranon (Figure 3).
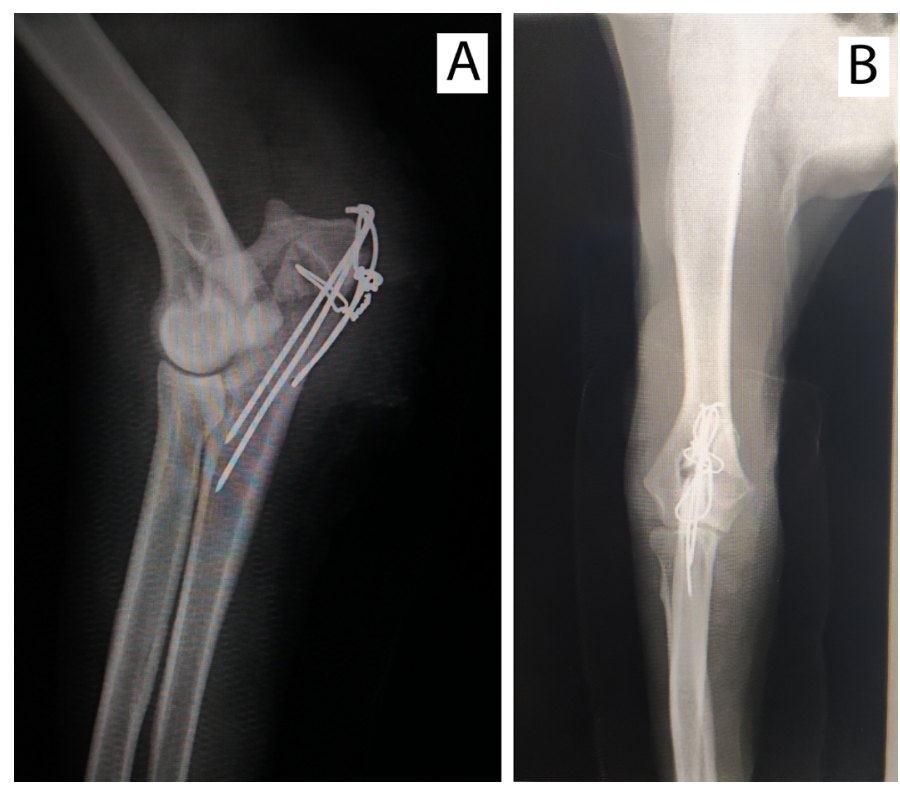

Figure 3: Postoperative mediolateral (A) and craniocaudal (B) radiographs showing two kirschner wires and tension band wire were placed across the olecranon fracture.

\section{Results and Discussion}

Fractures of the olecranon are usually seen in young dogs and different breeds of dog (Murthy et al., 2010; Nayak et al., 2012; Jones et al., 2015). Different techniques are used for olecranon fracture management in small animals. Out of all techniques, tension band wiring technique is most commonly used for olecranon fracture management. The optimal tension band wiring technique requires the $\mathrm{K}$-wires which provide more strength to the fractured fragments against muscles forces (Mullett et al., 2000; Grafinger et al., 2007). In the present study, internal fixation was performed using tension band wire and two K-wires which were perpendicular to the fracture and parallel to each other for management of transverse olecranon fracture. A major challenge is associated with open reduction and internal fixation of olecranon fractures at lateral approach where is the ability to fully visualize the articular margins of the fracture (Piermattei and Johnson, 2012). Tension band wiring technique requires proper size of $\mathrm{k}$-wires regarding the size of canine olecranon (Halling et al., 2003). The canine ulna is narrow proximally and marked concavity is present laterally (Grafinger et al., 2007). The management of olecranon fracture requires more attention and special care otherwise it may result in delayed non-union that trends to loss proper function of limb (Tyagi et al., 2002). Although, a separated bony part was observed intraoperatively at the proximal fracture end of olecranon, it was correctly fixed with wire at same fracture end. Fluoroscopy has been used intraoperatively assessing fracture reduction and proper placement of implants associated with olecranon fractures in dogs (Jones et al., 2015). Tension band wiring with plating technique provides adequate fixation and facilitates healing of fracture normally. But it is technically more difficult to perform considering the size of bone, time consuming and more degree of tissue manipulation (Tyagi et al., 2002).

The repairing of olecranon fracture is associated with high complication rate about $37 \%$ in dogs including osteomyelitis, loss of reduction, inappropriate placement and migration of $\mathrm{K}$-wires (Halling et al., 2002).In among them, $5 \%$ cases are found in migration of $\mathrm{K}$-wires during the early post-operative observation requiring to remove the implants (Halling et al., 2002). Unfortunately, on $30^{\text {th }}$ day of post-operative observation, $\mathrm{K}$-wires migration were found which protruded the skin but callus formation was observed between the fracture ends in radiograph. The small variations in technique that contribute significantly to the success or failure of the tension band wiring, and that the type of knot used may be of less importance than the surgeon's skill in its application (Grafinger et al., 2007). For avoidance of further complications, the implants were removed surgically and Modified Robert Jones bandage was applied in right forelimb 
for external support. The external support is needed in whole limb for immobilization, prevent the pull of tendon of triceps muscle, minimizes external trauma and facilitate early fracture healing (Murthy et al., 2010; Nayak et al., 2012). On 45 th day of clinical observations, the dog showed good weight bearing in right forelimb and walked normally (Figure 4). Even in heavy breed of dogs, tension band wiring in repairing of olecranon fractures provide rigid fixation and good clinical outcomes (Raghunath et al., 2008). If olecranon fractures are treated conservatively with external immobilization, the prognosis is unfavourable (O'Connor, 2005). In fact, complication of pin bending and breakage has been found when only intramedullary pinning has been used for repairing of olecranon fractures leading non-union to fibrous union (Denny, 1990).

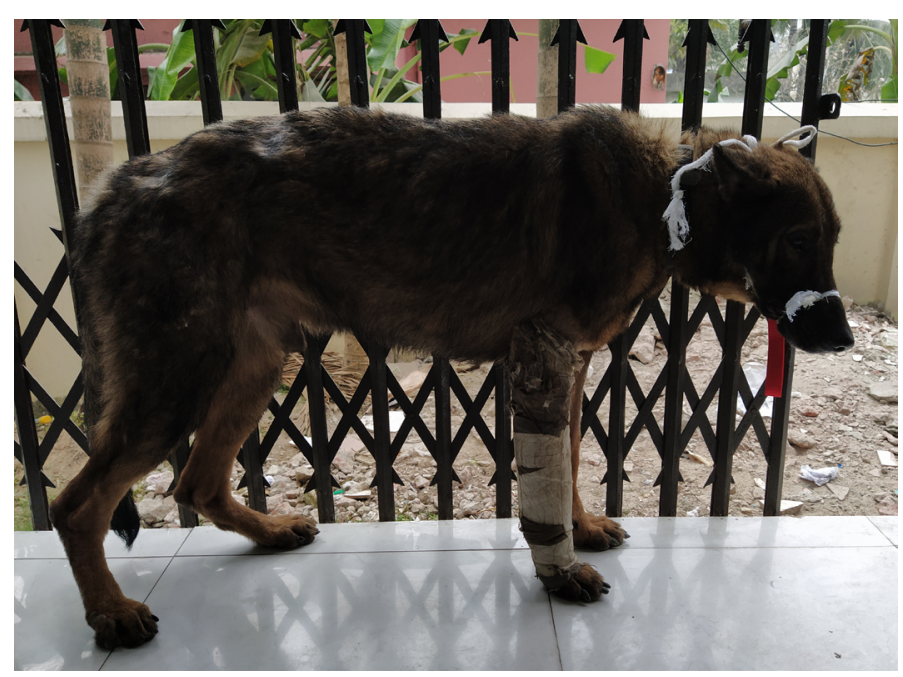

Figure 4: The dog was showing good weight bearing of the affected limb at 45 days of postoperative.

\section{Conclusions and Recommendations}

The clinical case study concluded that tension band wiring and pinning are good techniques to repair of olecranon fracture in dog. The fracture ends of the olecranon were completely healed within 45 days after operation. The dog showed good weight bearing with the affected limb and recovered normal posture.

\section{Acknowledgements}

The authors are grateful to the pet owner for well cooperation by providing valuable information and postoperative follow up until 45 days. The authors also grateful to Director of clinics, Chattogram Veterinary and Animal Sciences University for all kinds of technical supports to successfully perform this work.

\section{Novelty Statement}

Olecranon fracture in dog was corrected by pinning and tension band wiring technique that was first time used in Bangladesh.

\section{Author's Contribution}

Thomby Paul: Run the surgery, data acquisition and manuscript writing.

Sreekanta Biswas: Run the surgery.

Sabiha ZarinTasnim: Run the surgery.

Debashish Sarker: Run the surgery

Saroj Kumar Yadav: Run the surgery

Bhajan Chandra Das: Planning and run the surgery, manuscript writing and formatting.

\section{Conflict of interest}

The authors have declared no conflict of interest.

\section{References}

Denny H.R., 1990. Pectoral Limb fractures. In: Whittick, W.G., Canine Orthopedics, Lea and Febiger, Philadelphia, USA. $2^{\text {nd }}$ edn. pp. 373.

Evans, H.E. and de Lahunta, A., 2012. The skeleton. In: Evans, H.E. and de Lahunta, A., Miller's Anatomy of the Dog, Elsevier Saunders, USA. $4^{\text {th }}$ edn. pp. $134-136$.

Grafinger,M.S., Roe,S.C., Spodnick, G., and Smith, E.S., 2007. Biomechanical comparison of dual interlocking single loop and double loop tension band techniques to the classic $\mathrm{AO}$ tension band technique for repair of olecranon osteotomies in dogs. Vet. Surg., 36(2): 141-148. https://doi. org/10.1111/j.1532-950X.2007.00246.x

Halling, K.B., Lewis, D.D., and Cross, A.R., 2003. Biomechanical comparison of a circular external skeletal fixator construct to pin and tension band wire fixation for the stabilization of olecranon osteotomies in dogs: A cadaveric study. Vet. Surg., 32(4): 324-335. https://doi. org/10.1053/jvet.2003.50045

Halling, K.B., Lewis, D.D., Cross, A.R., Kerwin, S.C., Smith, B.A., and Kubilis, P.S., 2002. Complication rate and factors affecting outcome of olecranon osteotomies repaired with pin and tension-band fixation in dogs. Can. Vet. J., 43(7): 528.

Jones, S.C., Lewis, D.D. and Winter, M.D., 2015. Fluoroscopic-assisted olecranon fracture repair 
in three dogs. Case Rep. Vet. Med., 2015: 1-11. https://doi.org/10.1155/2015/542842

Muir, P., and Johnson, K.A., 1996. Fractures of the proximal ulna in dogs. Vet. Comp. Orthopaed. Traumatol., 9(2): 88-94. https:// doi.org/10.1055/s-0038-1632509

Mullett, J.H., Shannon, F., Nöel, J., Lawlor, G., Lee, T.C., and O'Rourke, S.K., 2000. K-wire position in tension band wiring of the olecranon. A comparison of two techniques. Injury, 31(6): 427-431. https://doi.org/10.1016/S00201383(00)00014-0

Murthy, K.S., Basha, K.M.A., Mahesh, V., Manjunatha, D.R., and Ranganath, L., 2010. Tension band wiring for avulsion fracture of olecranon in a dog. Vet. World, 3(11): 513-514.

Nayak, S., Behera, S.S., and Deshmukh, D.V., 2012. Internal and external fixation of olecranon fracture in a dog. Intas Polivet., 13(2): 388-390.
Newton, C.D. and Nunamaker, D.M., 1985. Textbook of small animal orthopaedics. Saunders, Philadelphia, USA.

O'Connor, J.J., 2005. Dollar's veterinary surgery. $4^{\text {th }}$ Edn. CBS Publishing, Delhi.

Piermattei, D.L. and Johnson, K.A., 2012. The forelimb. In: Piermattei, D.L. and Johnson, K.A., an atlas of surgical approaches to the bones and joints of the dog and cat, Saunders, Philadelphia, USA. $4^{\text {th }}$ edn. pp. 186-189.

Raghunath, M., Mohindroo, J., Chaudhary, M., Singh, N. and Sangwan, V., 2008. Tension band wiring for olecranon fracture repair in a dog.. Indian J. Vet. Surg., 29(2): 134.

Tyagi, S.P., Mogha, I.V., Singh, G.R., Aithal, H.P., and Amarpal. 2002. Management of olecranon fracture using different internal fixation techniques in dogs. Indian J. Vet. Surg., 23(2): 16-18. 